\title{
Primary Intestinal Follicular Lymphoma and Premature Atherosclerosis in a Japanese Diabetic Patient with Atypical Familial Partial Lipodystrophy
}

\author{
Masanori Iwanishi ${ }^{1}$, Ken Ebihara ${ }^{2}$, Toru Kusakabe ${ }^{3}$, Miki Washiyama ${ }^{1}$, Jun Ito-Kobayashi ${ }^{1}$, \\ Fumiyasu Nakamura ${ }^{4}$, Takeshi Togawa ${ }^{5}$, Yuki Ozamoto ${ }^{5}$, \\ Akeo Hagiwara $^{5}$ and Kazuwa Nakao ${ }^{3}$
}

\begin{abstract}
We experienced a case of primary intestinal follicular lymphoma and premature atherosclerosis in a diabetic patient with familial partial lipodystrophy (FPL) that was detected when the patient was evaluated for laparoscopic sleeve gastrectomy (LSG). As FPL is generally considered to be rare, FPL is often underdiagnosed, especially in obese patients. Therefore, the prevalence of FPL is higher than previous estimates. Our case illustrates that clinicians should perform screening for atherosclerosis and malignancy at the preoperative evaluation and may need to perform metabolic surgery earlier to prevent the development of excess truncal fat, complicated diabetes and atherosclerosis in patients with FPL.
\end{abstract}

Key words: familial partial lipodystrophy (FPL), premature atherosclerosis, metabolic surgery, primary intestinal follicular lymphoma

(Intern Med 53: 851-858, 2014)

(DOI: 10.2169/internalmedicine.53.1713)

\section{Introduction}

Lipodystrophy is classified as being either inherited or acquired and involving either generalized or partial loss of adipose tissue deposits (1-4). It is commonly associated with dyslipidemia, hepatic steatosis, and insulin-resistant diabetes. Familial partial lipodystrophy (FPL) is an autosomal dominant disorder, and mutations in the genes encoding laminin $\mathrm{A} / \mathrm{C}$, peroxisome proliferator-activated receptor- $\gamma$ (PPARG) and caveolin have been reported in patients with FPL. Familial partial lipodystrophy, Dunnigan variety (FPLD) is named after Dunnigan, who provided a detailed description of the syndrome (5). FPLD is associated with several mutations in the genes encoding lamin A/C. Many cases of FPL of European origin have been reported to be FPLD. FPL is often underdiagnosed due to the lack of detection of lipodystrophy (6-8). Systematic analyses of adipose tissue using dual energy X-ray absorptiometry (DEXA) or magnetic resonance imaging (MRI) scans are needed for diagnosis in some cases of FPL.

In this paper we present a case of FPL diagnosed on preoperative examination in a patient who was introduced to our hospital for laparoscopic sleeve gastrectomy (LSG). The patient had asymptomatic severe ischemic heart disease, severe narrowing of the right middle cerebral artery and primary intestinal follicular lymphoma. The patient canceled LSG for the treatment of follicular lymphoma and was at high operative risk due to severe atherosclerosis. This case illustrates that screening for atherosclerosis and malignancy is important in patients with FPL scheduled to undergo metabolic surgery.

\footnotetext{
${ }^{1}$ Department of Diabetes and Endocrinology, Kusatsu General Hospital, Japan, ${ }^{2}$ Institute for Advancement of Clinical and Translational Science, Kyoto University Hospital, Japan, ${ }^{3}$ Medical Innovation Center, Kyoto University Graduate School of Medicine, Japan, ${ }^{4}$ Department of Gastroenterology, Kusatsu General Hospital, Japan and ${ }^{5}$ Department of Digestive Surgery, Kusatsu General Hospital, Japan Received for publication September 5, 2013; Accepted for publication November 10, 2013 Correspondence to Dr. Masanori Iwanishi, masa-iwani@solid.ocn.ne.jp
} 


\section{Case Report}

The patient was a 48-year-old woman. At 33 years of age, she was diagnosed with diabetes by a diabetologist based on a high level of hemoglobin A1c (HbA1c) during pregnancy. She started insulin therapy immediately after diagnosis. Her diabetes remained uncontrolled (HbA1c level, 8.0-10.0\%), and she stopped the insulin injections temporally. At 44 years of age, her body weight was $75 \mathrm{~kg}$, suggesting that her body mass index (BMI) was 30.0. and her dose of insulin was increased to $104 \mathrm{U} /$ day $(1.4 \mathrm{U} / \mathrm{kg} /$ days $)$. She was admitted to our hospital for LSG at 48 years of age. Her height was $158 \mathrm{~cm}$ and her weight was $68.9 \mathrm{~kg}$, which suggested a body mass index of 27.6. The patient's history of body weight was as follows. When she was 9 years old, 15 years old, 20 years old, her weight was $30 \mathrm{~kg}, 60 \mathrm{~kg}$ and 65 $\mathrm{kg}$ (BMI 26), respectively. At 42 years of age, her weight was $89 \mathrm{~kg}$ (BMI 35.6). She had no history of autoimmune or infectious diseases and no habits of smoking or consuming alcohol. On admission, her HbA1c concentration was 9.3\% (Table 1), suggesting that her blood glucose control was poor, although the dose of insulin was increased to 39 U/day (0.57 U/kg/day). The serum TG level was $171 \mathrm{mg} / \mathrm{dL}$ and the high density lipoprotein (HDL) cholesterol level was $65 \mathrm{mg} / \mathrm{dL}$. She had no other abnormal endocrinological findings. The serum leptin level was $6.2 \mathrm{ng} / \mathrm{mL}$, and the serum adiponectin level was $2.3 \mu \mathrm{g} / \mathrm{mL}$. The urinary $\mathrm{C}$ peptide level was $5 \mu \mathrm{g} /$ day, suggesting severely decreased insulin secretion. The patient had mild non-proliferative diabetic retinopathy. Her total urinary albumin level was $22.5 \mathrm{mg} /$ day, which indicated the absence of diabetic nephropathy. Abdominal echo showed mild fatty liver, and the L/S ratio on CT value was 1.4. The platelet count and levels of serum albumin and hyaluronic acid and Type IV collagen were within the normal limits, which suggested that she did not have severe non-alcoholic steatosis hepatitis. She had noticed loss of subcutaneous fat over her forearm, lower limbs, and buttocks at 12 years of age. On admission, she exhibited loss of subcutaneous fat deposits in the forearm, lower limbs, and buttocks, with prominent lower limb musculature. She had excess fat deposition around the face, neck, and trunk, although that observed around the face and neck is covered with grey paper to protect her privacy (Fig. 1B). As shown in Fig. 1A, her mother had diabetes with a similar distribution of fat atrophy. Her two sisters and daughter did not have fat atrophy. An autosomal dominant pattern of inheritance was possible. The patient's mother was diagnosed with diabetes at 50 years of age, and died of pancreatic cancer at 79 years of age. The patient's father died of gastric cancer at 75 years of age and was not evaluated for fat atrophy or glucose tolerance. We assessed the distribution of body fat in the proband using DEXA and MRI studies. Thoracic (left panel) and abdominal MRI (right panel) revealed preservation of subcutaneous fat in the abdominal and thoracic regions in patient (Fig. 2A). MRI images obtained at
Table 1. Biochemical Data of the Patient

\begin{tabular}{lccc}
\hline Metabolic variables & Patient & Normal values \\
\hline Plasma glucose & 92 & $70-109$ & $(\mathrm{mg} / \mathrm{dL})$ \\
HbAlc & 9.3 & $4.6-6.2$ & $(\%)$ \\
Plasma CPR & 0.5 & $0.7-3.5$ & $(\mathrm{ng} / \mathrm{mL})$ \\
Serum triglycerides & 171 & $35-149$ & $(\mathrm{mg} / \mathrm{dL})$ \\
Serum cholesterol & 266 & $130-219$ & $(\mathrm{mg} / \mathrm{dL})$ \\
Serum HDL cholesterol & 65 & $40-83$ & $(\mathrm{mg} / \mathrm{dL})$ \\
Serum leptin & 6.2 & & $(\mathrm{ng} / \mathrm{mL})$ \\
Serum adiponectin & 2.3 & & $(\mu \mathrm{g} / \mathrm{mL})$ \\
Urinary CPR & 5 & & $(\mu \mathrm{g} / \mathrm{day})$ \\
\hline
\end{tabular}

the level of the gluteal fat revealed a marked loss of gluteal subcutaneous fat (Fig. 2B). Axial MRI performed at the level of the thigh (upper panel) and calf (lower panel) revealed a marked loss of subcutaneous fat in these regions (Fig. 2C). The patient had decreased amounts of subcutaneous fat, particularly in the antero-lateral and posterior thigh regions and exhibited the near complete loss of subcutaneous fat in the calf region. Axial MRI performed at the level of the forearm (lower panel) disclosed the almost complete absence of subcutaneous fat in the forearm, while axial MRI performed at the level of the arm (upper panel) demonstrated preservation of subcutaneous fat (Fig. 2D). The area of visceral fat at the umbilical level was $119.0 \mathrm{~cm}^{2}$. The regional and whole body adipose tissue distribution and body composition estimated with the DEXA scan are shown in Table 2. Compared to normal subjects, the patient had markedly lower levels of fat in her legs, with prominent accumulation of fat in the trunk. She appeared to have wellpreserved skeletal muscle. Therefore, we diagnosed her with FPL, based on her family history of lipodystrophy and, the onset of subcutaneous fat loss, and findings of the DEXA and MRI studies.

As the patient thought that her poor blood glucose control was caused by her rebound weight gain, she decided to undergo LSG. The surgeon asked us to control her blood glucose level and evaluate complications of diabetes. After we diagnosed the patient with FPL, we performed magnetic resonance angiography (MRA) to screen for atherosclerosis in the cervical and cerebral arteries. We also performed multidetector-row computed tomography (MDCT) containing 64 slices with a high spatial resolution of $0.6 \mathrm{~mm}$ to screen for atherosclerosis in the coronary arteries. The MRA findings showed severe narrowing at the end of the right internal carotid artery (Fig. 3A) with narrowing of the left internal carotid artery (Fig. 3B). The narrowing in the proximal region of the right middle cerebral artery and left internal carotid artery is indicated by arrows. MDCT showed coronary calcification in the right coronary artery (Fig. 3C) and left coronary artery (Fig. 3D). The coronary calcification was more severe in the left anterior descending artery and left circumflex coronary artery than in the right coronary artery. MDCT showed $75 \%$ narrowing of \#3, the distal right coronary and \#9, first diagonal branch, and 50\% narrowing of \#6, \#7 proximal and middle left anterior descend- 
A

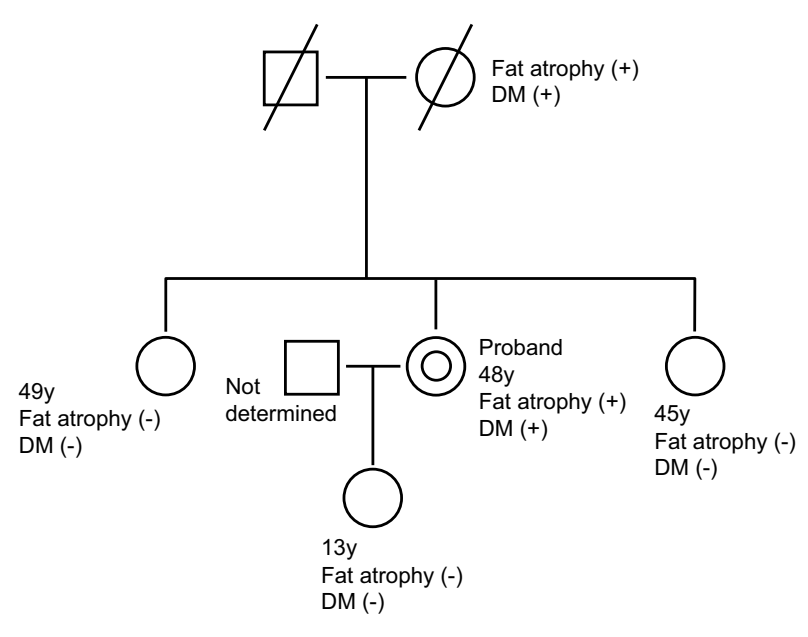

B

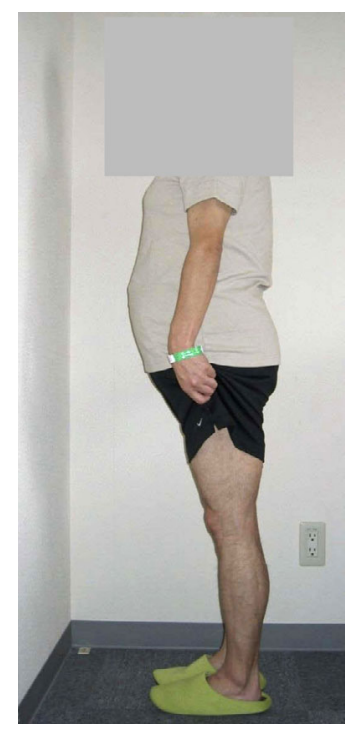

Figure 1. A: The pedigree of a Japanese family with lipodystrophy. While the proband and her mother exhibited marked loss of subcutaneous fat in the lower limbs and buttocks, her two sisters and daughter did not have lipodystrophy. The proband and her mother had diabetes mellitus. B: Phenotypic features of the proband are shown in the right panel. Note the prominent musculature of the lower limbs as well as the preservation of abdominal fat with loss of fat in the lower limbs and gluteal tissues. Excess fat deposition around the neck is covered with grey paper to protect the patient's privacy. She had fat atrophy in the forearm, with conservation of subcutaneous fat in the arm.

A

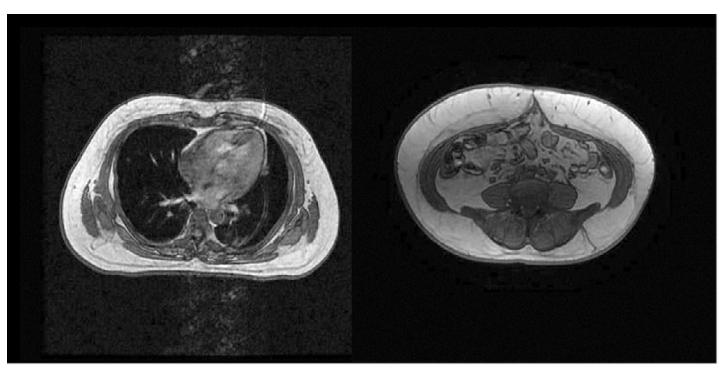

B

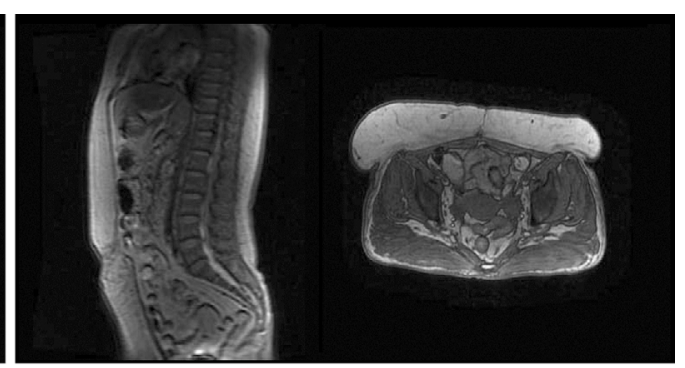

C

D
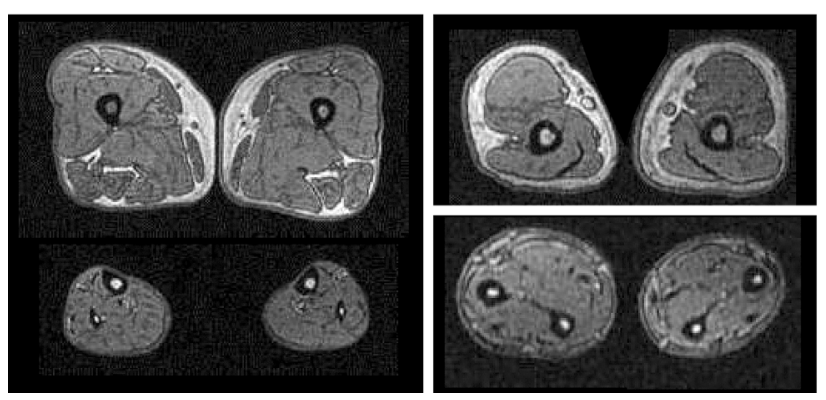

Figure 2. Magnetic resonance imaging (MRI) in the proband. A: Thoracic MRI images obtained at the level of the fourth thoracic vertebrae (left panel) and abdominal MRI images obtained at the umbilical level (right panel) show preservation of subcutaneous fat in the thoracic and abdominal regions. B: T1-weighted MRI image obtained at the level of the gluteal fat indicated a striking loss of gluteal subcutaneous fat. C: MRI images obtained at the level of the thigh (upper panel) and calf (lower panel) in the patient revealed the nearly complete absence of subcutaneous fat. The patient had a decreased amounts of subcutaneous fat, particularly in the antero-lateral and posterior thigh region, and exhibited the near complete loss of subcutaneous fat in the calf region. D: MRI images obtained at the level of the arm (upper panel) and forearm (lower panel) revealed marked loss of subcutaneous fat in the forearm, with preservation of fat in one arm. 
Table 2. Body Composition as Determined by DEXA Scan in the Patient

\begin{tabular}{|c|c|c|}
\hline & Patient & mean values (in control subjects) \\
\hline Height $(\mathrm{cm})$ & 158.0 & $156.1 \pm 4.9$ \\
\hline Body weight (kg) & 68.9 & $55.1 \pm 6.7$ \\
\hline $\operatorname{BMI}\left(\mathrm{kg} / \mathrm{m}^{2}\right)$ & 27.6 & $22.7 \pm 3.0$ \\
\hline Age (years) & 48 & $45.1 \pm 2.8$ \\
\hline \multicolumn{3}{|l|}{ 1) Fat (\%) } \\
\hline whole body & 28.2 & $30.3 \pm 6.2$ \\
\hline one arm & 31.9 & $26.9 \pm 7.3$ \\
\hline one leg & 12.1 & $33.2 \pm 5.7$ \\
\hline trunk & 36.1 & $28.4 \pm 7.7$ \\
\hline \multicolumn{3}{|l|}{ 2) Fat Mass (kg) } \\
\hline whole body & 18.2 & $16.8 \pm 5.2$ \\
\hline one arm & 0.96 & $0.7 \pm 0.3$ \\
\hline one leg & 1.0 & $3.5 \pm 1.0$ \\
\hline trunk & 13.3 & $7.1 \pm 2.8$ \\
\hline \multicolumn{3}{|l|}{ 3) Lean Mass (kg) } \\
\hline whole body & 44.1 & $35.3 \pm 3.1$ \\
\hline one arm & 1.9 & $1.7 \pm 0.2$ \\
\hline one leg & 6.9 & $6.6 \pm 0.9$ \\
\hline trunk & 22.8 & $16.3 \pm 1.3$ \\
\hline
\end{tabular}

ing branch and \#13 distal left circumflex branch (data not shown). These data suggested that the patient had premature severe atherosclerosis

Computed tomography (CT) scans of the abdomen demonstrated swelling of the para-aortic lymph node, $(25 \mathrm{~mm}$ in diameter) (Fig. 4A). An 18F-fluorodeoxyglucose position emission tomography scan (PET) showed abnormal accumulation of $18 \mathrm{~F}$-fluorodeoxyglucose in the area of swelling in the para-aortic lymph node detected on CT (Fig. 4B). Colonoscopey revealed whitish polyps in the terminal ileum (Fig. 4C). Indigo carmine spraying visualized these microsurface structures more clearly (Fig. 4D). Based on these endoscopic features, a diagnosis of ileal follicular lymphoma was highly suspected. The biopsy samples contained one large lymphoid follicle in the ileal mucosa (Fig. 5A) comprised of small- to medium-sized lymphoid cells that had also infiltrated into the villi (Fig. 5B). Immunostaining showed the lymphoid cells to be positive for CD20, CD10, and BCL2 (Fig. 5C, 5D, 5E), and negative for CD3 (data not shown). Consequently, the patient was diagnosed with primary intestinal follicular lymphoma, associated with paraaortic lymphatic metastasis.

We prescribed pioglitazone $(7.5 \mathrm{mg} /$ day $)$, because thiazolidinediones have been reported to effective in achieving glycemic control in patients with FPL. We examined the patient's response to pioglitazone by monitoring the $\mathrm{HbA} 1 \mathrm{c}$ levels and changes in her biochemical data and, body weight, during treatment. After three months of treatment, the HbA1c level decreased from $9.3 \%$ to $7.8 \%$, then remained at approximately $7.4 \%$. After three months, her body weight increased from 68.9 to $71.0 \mathrm{~kg}$. In addition, we evaluated changes in the leptin and adiponectin levels during pioglitazone treatment. After nine months of treatment, the serum adiponectin level increased from 2.3 to $4.0 \mu \mathrm{g} / \mathrm{mL}$ and the serum leptin level increased from $6.2 \mathrm{ng} / \mathrm{mL}$ to 10.6 $\mathrm{ng} / \mathrm{mL}$.

We examined the sequences of the entire coding region and exon-intron boundary regions of the LMNA and PPARG genes, which are known to be associated with FPL; however, we found no mutations in these genes in the proband. We also examined the deletions of the entire coding region of the LMNA, PPARG, CAV3, ZMPSTE24 and MYOT genes, using multiplex ligation-dependent probe amplification (MLPA) analyses, and also found no deletions in these genes in the proband.

The patient was ultimately transferred to another hospital for treatment of follicular lymphoma and coronary artery stenosis.

\section{Discussion}

Three previous reports have shown Roux-en-Y gastric bypass (RYGBP) to be effective in improving metabolic disturbances in women with FPL (9-11). Recently, Ciudin reported the first case of FPLD in which RYGBP was found to be an effective treatment for both metabolic disturbances, and abnormal fat accumulation in a patient with Dunnigantype FPLD (9). Therefore, metabolic surgery appears to be effective to in improving metabolic disturbances and the development of excess fat in obese patients with FPL. However, we canceled LSG in the present cases, because the patient had severe atherosclerosis and primary intestinal follicular lymphoma.

FPL is often underdiagnosed for the following reasons. First, as FPLD is a rare disorder with an estimated prevalence of 1 in 20,-0000 (12), FPL is generally considered to be rare. Second, as some patients with FPL are obese with excess fat in the trunk and fat atrophy in the extremities, they may often be misdiagnosed with obesity. Therefore, it is difficult to notice lipodystrophy in obese FPL patients without conducting a systematic analysis of the adipose tissue distribution (7). In one study of over 5,000 Dutch patients with diabetes treated at three outpatient clinics at which two screening criteria were applied (body mass index $27 \mathrm{~kg} / \mathrm{m}^{2}$ and use of $>100$ units of insulin/day), five of the patients were eventually diagnosed with FPL (8). Although the prevalence of FPL in patients with Type 2 diabetes has not been reported, we have diagnosed approximately 30 cases of FPL, using DEXA and MRI among outpatients at Kusatsu General Hospital since August 2003 (unpublished data). Therefore, clinicians should keep in mind that FPL may have a greater prevalence than previously thought. Recently, the American Association of Clinical Endocrinologists (AACE) provided consensus recommendations for the detection of lipodystrophy for clinical practitioners (6). This document attempts to educate clinicians about lipodystrophy, increase awareness of its presence and, improve detection of underdiagnosed cases. 

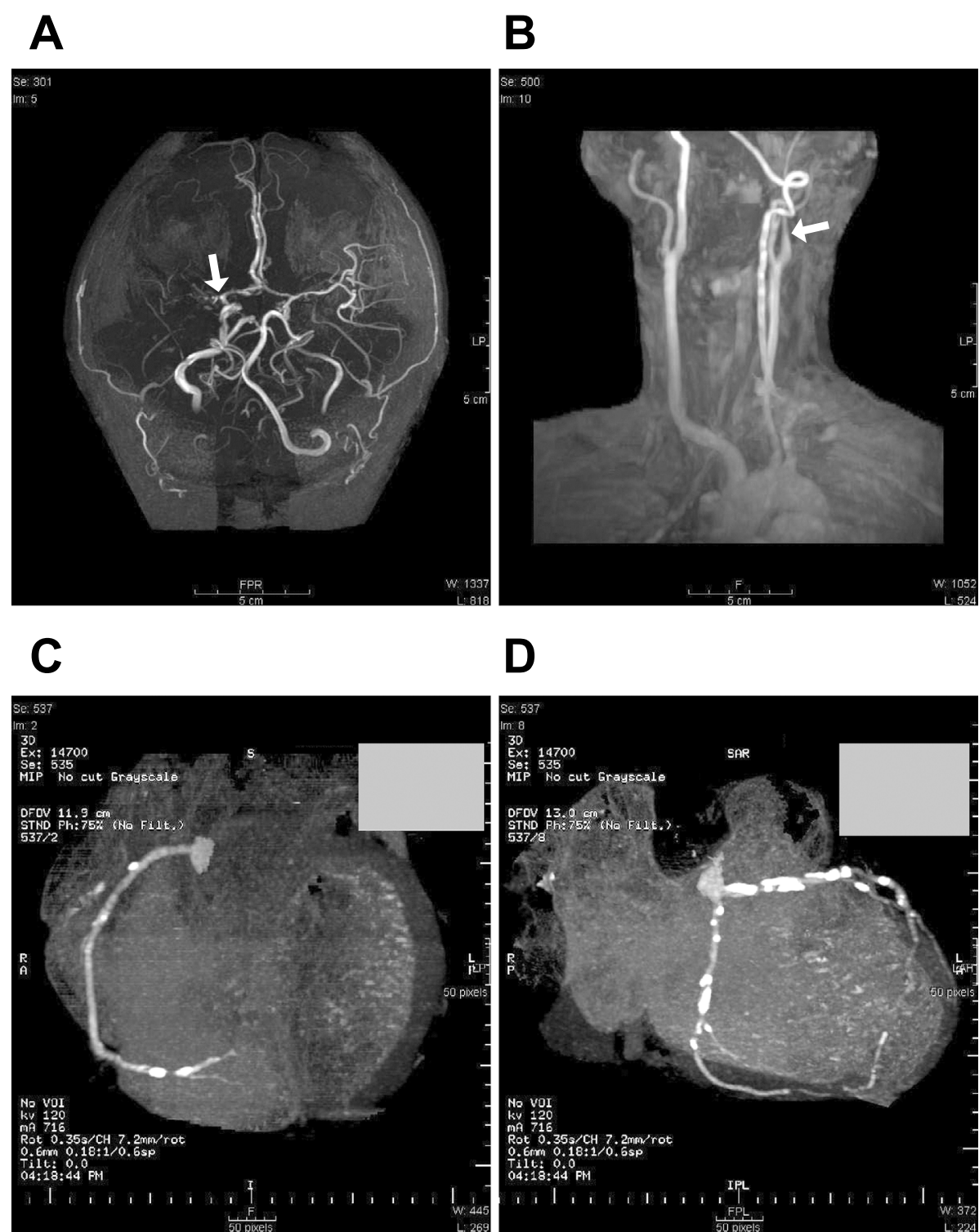

D

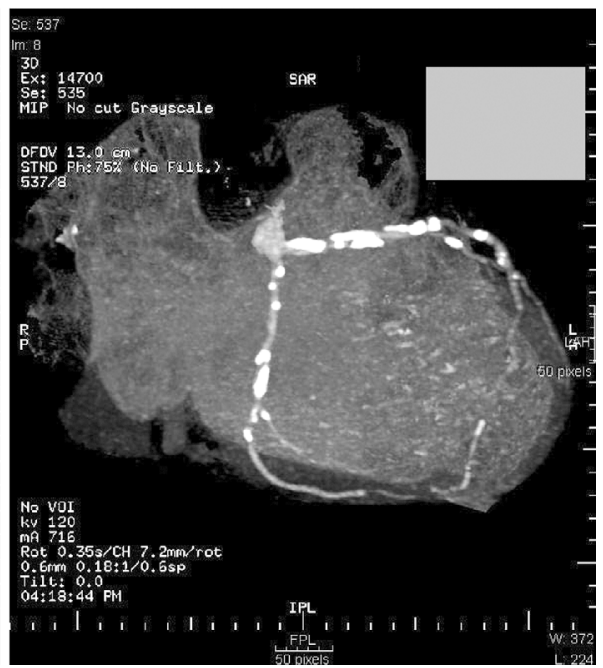

Figure 3. Brain magnetic resonance angiography (MRA) showed severe narrowing at the end of the right internal carotid artery (A) and narrowing of the left internal carotid artery (B). The narrowing in the proximal region of the right middle cerebral artery and left internal carotid artery is indicated by arrows. MDCT showed coronary calcification in the right coronary artery (C) and left coronary artery (D). The coronary calcification was more severe in the left anterior descending artery and left circumflex coronary artery than in the right coronary artery.

In some cases of FPL, the lipodystrophy is confined to the limbs, with sparing of the face and trunk (atypical FPL), whereas the trunk is also affected with sparing of the face and vulva in other cases (typical FPLD). It has been reported that the phenotypes of FPL, including FPLD are diverse (13). Mutations in exon 11 of LMNA affect the globular domain specific to the lamin A isoform, while mutations in exon 8 affect the globular domain of both splice forms, lamin A and lamin C. Garg observed a milder form of lipodystrophy as well as less severe metabolic complications in atypical FPL patients with mutations in exon 11 of LMNA in comparison to that observed in patients with mutations in exon 8. Therefore, the severity of mutations in candidate genes appears to determine the phenotype, ie.,typi- cal FPLD or atypical FPL. Therefore, it is conceivable that the patient in the present case exhibited atypical FPL with mild impairment of adipocyte differentiation caused by a novel mild gene mutation.

As the amount of adipose tissues in non-obese patients with FPL is less than that observed in non-obese healthy subjects, the levels of serum leptin are lower in non-obese patients with FPL and FPLD than in non-obese healthy subjects (14). More leptin may be released from increased fat tissues; therefore, the levels of serum leptin in some obese patients with FPL are higher than those observed in nonobese patients with FPL (unpublished data). Low levels of serum leptin in patients with FPL may decrease satiety, thereby causing overeating $(15,16)$. Patients with milder 
A

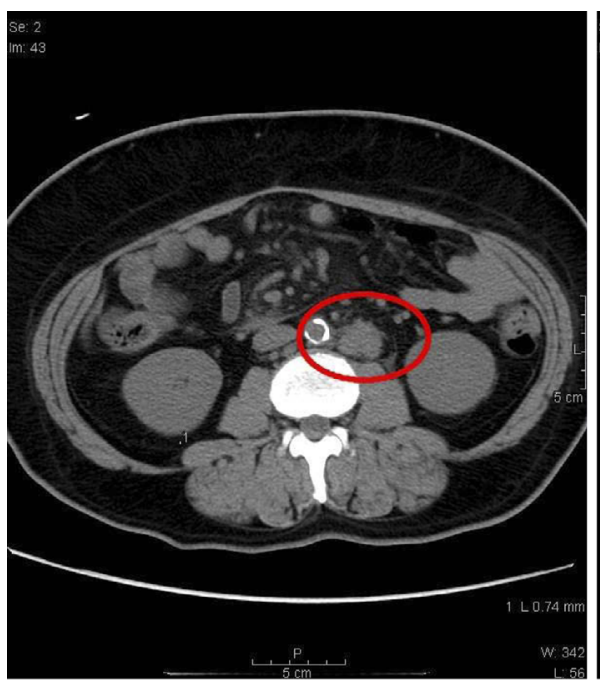

C

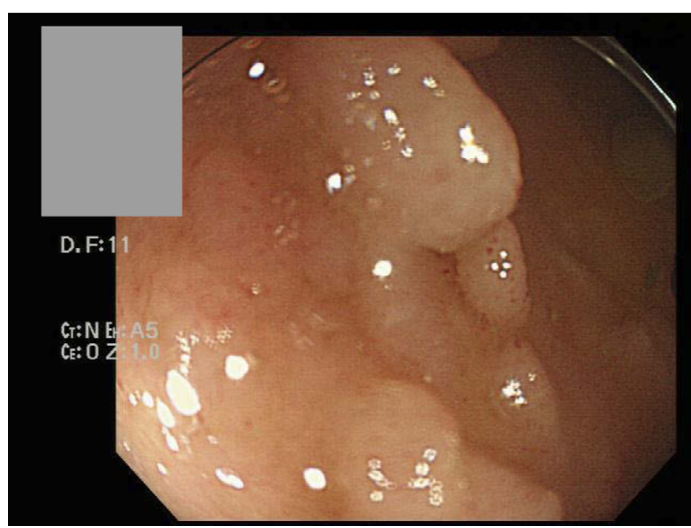

B

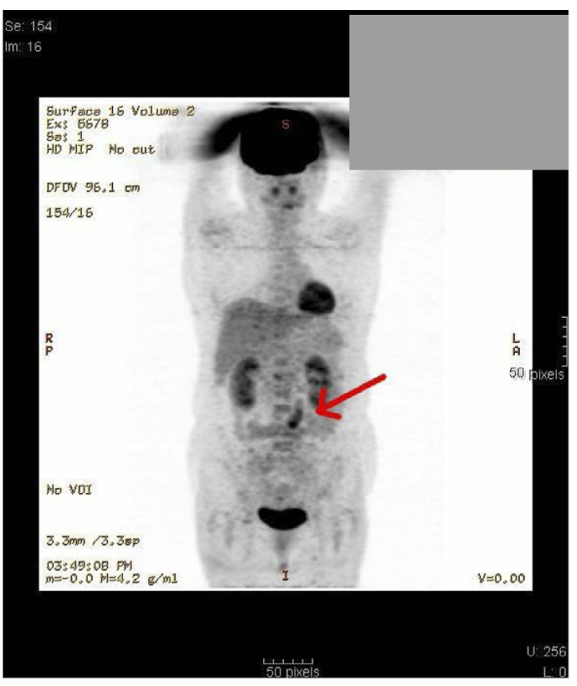

D

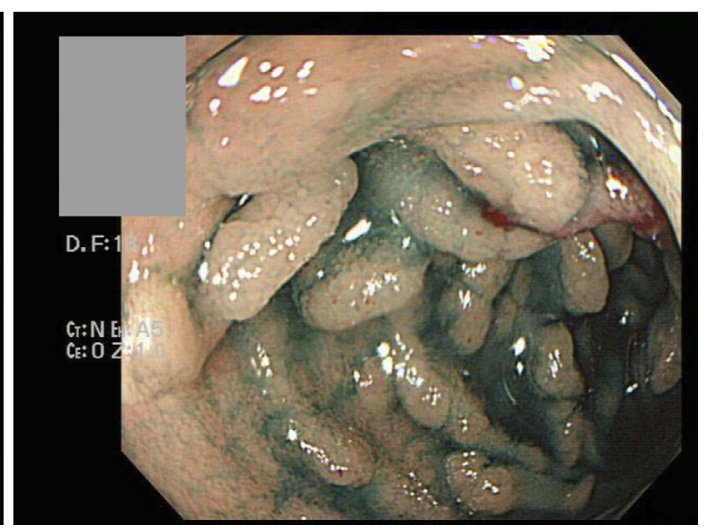

Figure 4. Computed tomography (CT) scans of the abdomen detected swelling of the para-aortic lymph node, indicated by the red circle (A). An 18F-fluorodeoxyglucose position emission tomography scan (PET) showed abnormal accumulation of $18 \mathrm{~F}$-fluorodeoxyglucose in the area of swelling in the para-aortic lymph node on $\mathrm{CT}$, indicated by the red arrow (B). Colonoscopey revealed whitish polyps in the terminal ileum (C). Indigo carmine spraying visualized these microsurface structures more clearly (D).

lipodystrophy, who have low levels of serum leptin, tend to be obese. While the mean serum leptin level was $11.1 \pm 2.0$ (mean $\pm \mathrm{SD}$ ) in 28 women with normal glucose tolerance (age $58 \pm 2 \mathrm{yr}$, BMI $24.0 \pm 1.0 \mathrm{~kg} / \mathrm{m}^{2}$ ) (17), the current patient's serum leptin level was $6.2 \mathrm{ng} / \mathrm{mL}$, suggesting that her serum leptin level was relatively low. This may have caused the truncal obesity observed in this case. Therefore, leptin replacement may be effective in preventing the accumulation of excess fat in some cases of FPL $(18,19)$. Gavrilova O reported that surgical implantation of adipose tissue reverses diabetes in lipoatrophic mice (20). Therefore, transplantation of adipocytes derived from human iPS cells in patients with FPL is a potential alternative therapy to prevent excess fat accumulation and diabetes.

A low levels of serum adiponectin associated with the development of several cancers, including colon cancer (21-23). However, the mechanisms underlying the association between a decreased level of plasma adiponectin and carcinogenesis remains to be fully understood. Mutoh demonstrated that mice with disruptions in plasma adiponectin develop more intestinal tumors (23). Therefore, it is possible that FPL is associated with malignancy, as affected patients have low levels of serum adiponectin. Indeed, Matsumoto reported a high prevalence of cancer of the digestive organs, including colon cancer and gastric cancer, in a family with congenital generalized lipodystrophy (24). Franceschini also reported pre-B acute lymphoblastic leukemia in a patient with partial lipodystrophy (25), while Shinkawa reported ovarian carcinoma in a patient with congenital generalized lipodystrophy (26).

We detected intestinal follicular lymphoma in this case. It has been reported that there is a possibility that obesity results in a pathological state of chronic low level inflammation and altered immune responses that may influence the B and $\mathrm{T}$ lymphocyte function and thus the development of non-Hodgkin lymphoma (NHL) (27). It has also been re- 
A

B
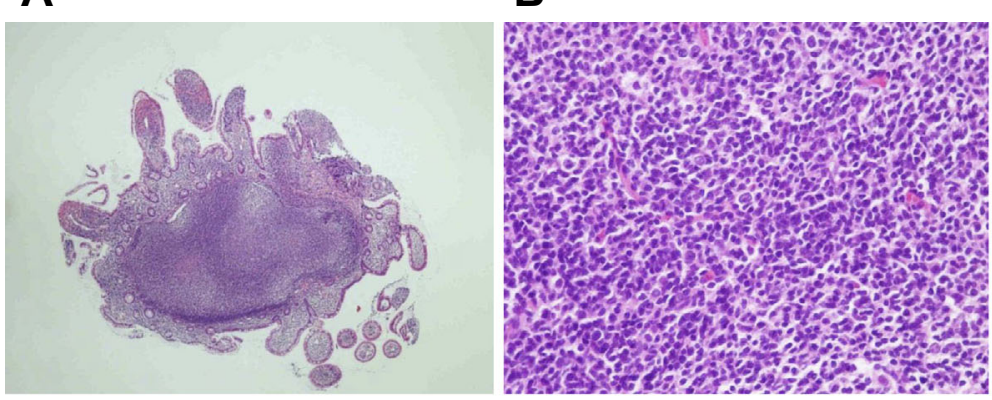

C

D

\section{E}
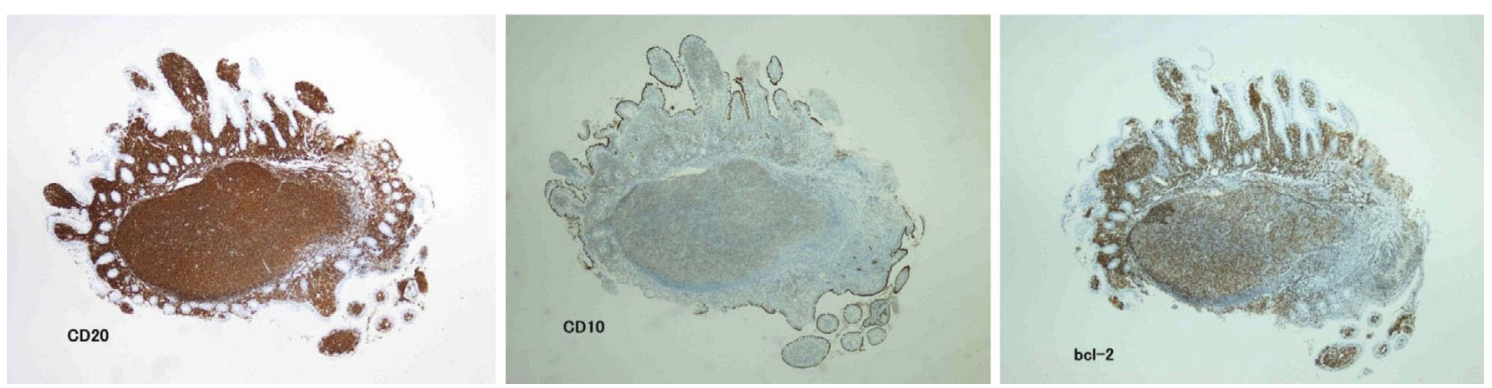

Figure 5. The biopsy samples contained one large lymphoid follicle in the ileal mucosa (A) comprised of small- to medium-sized lymphoid cells that had also infiltrated into the villi (B). Immunostaining showed the lymphoid cells to be positive for CD20, CD10 and, BCL2 (C, D, E).

ported that chronic low level inflammation is detected in adipose tissues in patients with FPL and $\operatorname{FPLD}(28,29)$. Conroy reported that the levels of serum IL-10 was found to be positively associated, while the level of serum leptin was inversely associated, with the prevalence of follicular lymphoma in a nested case-control analysis (27). Leptin participates in the inflammation response and enhances B cell survival. Therefore, chronic low level inflammation and a relatively low levels of leptin have been associated with follicular lymphoma in this case.

The age of onset of diabetes mellitus in the present patient was 33 years, which is relatively early compared to that observed in most patients with type 2 diabetes mellitus. At 48 years of age, the present patient exhibited severe narrowing at the end of the right internal carotid artery, left cervical artery and coronary arteries, which suggested that she had severe premature atherosclerosis. Her serum adiponectin level was $2.3 \mu \mathrm{g} / \mathrm{mL}$. These data suggested that the patient's serum adiponectin level was relatively low compared to that observed in 28 women with normal glucose tolerance (17). The level of adiponectin may also have had an effect on atherosclerosis in the present case. Therefore, because our patient was a woman with FPL and exhibited clustering of atherogenic risk factors, such as diabetes mellitus, hypertriglyceridemia and a low serum levels of adiponectin, it is conceivable that atherogenic risk factors and other factors, including unidentified causative genes affect the development of atherosclerosis, as we previously reported (30-35).

Pioglitazone decreased the levels of HbA1C in the present case, with a small increase in the level of serum adi- ponectin, although the effects of thiazolidinediones on glycemic control and the serum adiponectin levels appear to vary in FPL patients (34-36). Although why pioglitazone did not significantly increase the serum adiponectin level in the present patient is unknown, it appears that we could not expect preventive effects on atherosclerosis via an increased levels of serum adiponectin induced by pioglitazone in this case.

A sequencing and deletion analysis of the candidate genes LMNA and PPARG which are known to be associated with FPL, revealed no genetic abnormalities in the present case. Genetic testing is confirmatory in only approximately $50 \%$ of FPL cases, (12) and this case may have involved a novel gene.

In conclusion, we herein described a case of premature atherosclerosis and primary intestinal follicular lymphoma in a Japanese diabetic patient with atypical FPL and hypertriglyceridemia. Although it has been reported that metabolic surgery is effective in reducing body weight in patients with FPL, this case illustrates that clinicians should perform screening for atherosclerosis and malignancy during preoperative evaluations and may need to perform metabolic surgery earlier to prevent the development of excess truncal fat, complicated diabetes and atherosclerosis in patients with FPL.

\section{The authors state that they have no Conflict of Interest (COI).}

\section{Acknowledgement}

We thank Dr. Takashi Shiokawa (Tanita Inc., Tokyo, Japan) for providing the DEXA data of 55 healthy women between the 
ages of 40 and 49 years and 72 healthy men between the ages of 20 and 29 years.

\section{References}

1. Garg A. Acquired and inherited lipodystrophies. N Engl J Med 350: 1220-1234, 2004.

2. Agarwal AK, Garg A. Genetic disorders of adipose tissue development, differentiation and death. Annu Rev Genomics Hum Genet 7: 175-199, 2006.

3. Agarwal AK, Garg A. Genetic basis of lipodystrophies and management of metabolic complications. Annu Rev Med 57: 297-311, 2006.

4. Garg A. Lipodystrophies: Genetic and acquired body fat disorders. J Clin Endocrinol Metab 96: 3313-3325, 2011.

5. Dunnigan MG, Cochrane MA, Kelly A, Scott JW. Familial lipoatrophic diabetes with dominant transmission. Quarterly Journal of Medicine 169: 33-48, 1974.

6. Handelsman Y, Oral EA, Bloomgarden ZT, et al. The clinical approach to the detection of lipodystrophy an aace consensus statement. Endocr Pract 19: 107-116, 2013.

7. Vantyghem MC, Balavoine AS, Douillard C, et al. How to diagnose a lipodystrophy syndrome. Annales of Endocrinologie 73: 170-189, 2012.

8. Visser ME, Kropman E, Kranendonk ME. Characterisation of non-obese diabetic patients with marked insulin resistance identifies a novel familial partial lipodystrophy-associated PPARgamma mutation (Y151C). Diabetogia 54: 1639-1644, 2011.

9. Ciudin A, Baena-Fustegueras JA, Fort JM, Encabo G, Lecube A. Successful treatment of the Dunnigan-type familial partial lipodystrophy with Roux-en-Y gastric bypass. Clinical Endocrinol 75: 403-404, 2011.

10. Utzschneider KM, Trence DL. Effectiveness of gastric bypass surgery in a patient with familial partial lipodystrophy. Diabetes Care 6: 1380-1382, 2006.

11. McGrath NM, FRACP M, Krishna G; MBChB, FRACS. Gastric bypass for insulin resistance due to lipodystrophy. Obes Surg 16: 1542-1544, 2006.

12. Joy T, Kennedy BA, Al-Attar S, Rutt BK, Hegele RA. Predicting abdominal adipose tissue among women with familial partial lipodystrophy. Metabolism 58: 828-834, 2009.

13. Mory PB, Crispim F, Freire MB, et al. Phenotypic diversity in patients with lipodystrophy associated with LMNA mutations. Eur J Endocrinol 167: 423-431, 2012.

14. Haque WA, Shimomura I, Matsuzawa Y, Garg A. Serum adiponectin and leptin levels in patients in patients with lipodystrophies. J Clin Endocrinol Metab 87: 2395-2398, 2002.

15. McDuffie JR, Riggs PA, Calis KA, et al. Effects of exogenous leptin on satiety and satiation in patients with lipodystrophy and leptin insufficieny. J Clin Endocrinol Metab 89: 4258-4263, 2004.

16. Aotani D, Ebihara K, Sawamoto N, et al. Functional magnetic resonance imaging analysis of food-related brain activity in patients with lipodystrophy undergoing leptin replacement therapy. J Clin Endocrinol Metab 97: 3663-3671, 2012.

17. Hotta K, Funahashi T, Arita Y, et al. Plasma concentrations of a novel, adipose-specific protein, adiponectin, in type 2 diabetic patients. Arterioscler Thromb Vasc Biol 20: 1595-1599, 2000.

18. Ebihara K, Kusakabe T, Hirata M, et al. Efficacy and safety of leptin-replacement therapy and possible mechanisms of leptin actions in patients with generalized lipodystrophy. J Clin Endocrinol Metab 92: 532-541, 2007.

19. Moon HS, Dalamaga M, Kim SY, et al. Leptin's role in lipodys- trophic and nonlipodystrophic insulin-resistant and diabetic individuals. Endocrine Reviews 34: 1-36, 2013.

20. Gavrilova O, Marcus-Samuels B, Graham D, et al. Surgical implantation of adipose tissue reverses diabetes in lipostrophic mice. J Clin Invest 105: 271-278, 2000.

21. Dalamaga M, Diakopoulos KN, Mantzoros CS. The role of adiponectin in cancer: A review of current evidence. Endocrine Reviews 33: 547-594, 2012.

22. Bao Y, Giovannucci EL, Stampfer MJ, et al. A prospective study of plasma adiponectin and pancreatic cancer risk in five US cohorts. J Natl Cancer Inst 105: 95-103, 2013.

23. Mutoh M, Teraoka N, Takasu S, et al. Loss of adiponectin promotes intestinal carcinogenesis in min and wild-type mice. Gastroenterology 140: 2000-2008, 2011.

24. Matsumoto N, Suehio T, Nishiyama M, et al. A family with lipoatrophic diabetes, high frequency of type 2 diabetes mellitus nd cancer in the digestive organs. J. Japan Diab. Soc 43: 51-56, 2000.

25. Franceschini P, Barisone E, Signorile F, et al. Pre-B acute lymphoblastic leukemia in a patient with partial lipodystrophy and acanthosis nigricans. Panminerva Med 37: 248-251, 1995.

26. Shinkawa K, Nishikawa H, Mori T, Kawai H, Yoshida Y. Case of congenital generalized lipodystrophy associated with papillary serous cysadenocarcinoma. Horumon To Rinsho 25: 888-895, 1977 (in Japanese).

27. Conroy SM, Maskarinec G, Morimoto Y, et al. Non-Hodgkin lymphoma and circulating markers of inflammation and adiposity in a nested case-control study: The multiethnic cohort. Cancer Epidemiol Biomarkers Prev 1: 1-24, 2013.

28. Bereziat V, Cervera P, Le Dour C, et al; the Lipodystrophy Study Group. LMNA mutations induce a non-inflammatory fibrosis and a brown fat-like dystrophy of enlarged cervical adipose tissue. Am J Pathol 179: 2443-2453, 2011.

29. Gandotra S, Dour CL, Bottomley W, et al. Perilipin deficiency and autosomal dominant partial lipodystrophy. N Engl J Med 364: 740-748, 2011.

30. Garg A. Gender differences in the prevalence of metabolic complications in familial partial lipodystrophy (Dunnigan variety). J Clin Endocrinol Metab 85: 1776, 2000.

31. Hegele RA. Premature atherosclerosis associated with monogenic insulin resistance. Circulation 103: 2225-2229, 2001.

32. Bidault G, Garcia M, Vantyghem M-C, et al. Lipodystrophy-linked LMNA p.R482W mutation induces clinical early atherosclerosis and in vitro endothelial dysfunction. Arterioscler Thromb Vasc Biol 33: 1-10, 2013.

33. Auclair M, Vigouroux C, Boccara F, et al. Peroxisome proliferator-activated receptor- $\gamma$ mutations responsible for lipodystrophy with severe hypertension activate the cellular reninangiotensin system. Arterioscler Thromb Vasc Biol 33: 829-838, 2013.

34. Iwanishi M, Ebihara K, Kusakabe T, et al. Premature atherosclerosis in a japanese diabetic patient with atypical familial partial lipodystrophy and hypertriglyceridemia. Intern Med 51: 25732579, 2012.

35. Iwanishi M, Ebihara K, Kusakabe T, et al. Clinical characteristics and efficacy of pioglitazone in a Japanese diabetic patient with an unusual type of familial partial lipodystrophy. Metabolism 58: 1681-1687, 2009.

36. Arioglu EA, Duncan-Morin J, Sebring N, et al. Efficacy and safety of troglitazone in the treatment of lipodystrophy syndromes. Ann Intern Med 133: 263-274, 2000.

(C) 2014 The Japanese Society of Internal Medicine http://www.naika.or.jp/imonline/index.html 